\title{
Cosmological Implications of Dynamical Supersymmetry Breaking*
}

\author{
Tom Banks \\ Department of Physics and Astronomy \\ Rutgers University \\ Piscataway, NJ 08855-0849 \\ David B. Kaplan ${ }^{\dagger}$ and Ann E. Nelson \# \\ Department of Physics \\ 9500 Gilman Drive 0319 \\ University of California, San Diego \\ La Jolla, CA 92093-0319
}

\begin{abstract}
We provide a taxonomy of dynamical supersymmetry breaking theories, and discuss the cosmological implications of the various types of models. Models in which supersymmetry breaking is produced by chiral superfields which only have interactions of gravitational strength (e.g. string theory moduli) are inconsistent with standard big bang nucleosynthesis unless the gravitino mass is greater than $\mathcal{O}(3) \times 10^{4} \mathrm{GeV}$. This problem cannot be solved by inflation. Models in which supersymmetry is dynamically broken by renormalizable interactions in flat space have no such cosmological problems. Supersymmetry can be broken either in a hidden or the visible sector. However hidden sector models suffer from several naturalness problems and have difficulties in producing an acceptably large gluino mass.
\end{abstract}

hep-ph@xxx/9308292, UCSD/PTH 93-26, RU-37

July 1993

\footnotetext{
* This work is supported in part by funds provided by the U. S. Department of Energy (D.O.E.) under contracts \#DOE-FG03-90ER40546and DE-FG0590ER40559, by the National Science Foundation under grant PHY-9057135, by the Sloan Foundation and by the Texas National Research Laboratory Commission under grant \#RGFY93-206.

$\dagger$ National Science Foundation Young Investigator Award.

\# Alfred P. Sloan Foundation Research Fellowship.
} 


\section{Introduction}

From a practical point of view, the primary allure of supersymmetric field theories lies in their promise to solve the gauge hierarchy problem. Supersymmetry succeeds in explaining why $M_{W} / M_{P}$ is not $\mathcal{O}(1)$ by eliminating all quadratic divergences. However, an explanation for the observed value $M_{W} / M_{P}=10^{-17}$ cannot be furnished by supersymmetry itself, but only by some theory for dynamical supersymmetry breaking, in which the scale of the weak interactions arises from the Planck scale and dimensionless coupling constants through dimensional transmutation 1 团. In dynamical supersymmetry (SUSY) breakdown scenarios, SUSY is unbroken to all orders in perturbation theory but broken by nonperturbative effects proportional to $e^{-a / g^{2}}$, where $a$ is a number of order $4 \pi^{2}$ and $g$ is a gauge coupling constant. At present, there does not exist a unique compelling scenario for dynamical supersymmetry breaking which is compatible with standard particle phenomenology, and so our present understanding of the supersymmetric resolution of the hierarchy problem is incomplete. The purpose of the present paper is not to resolve this problem, but to delineate the options for dynamical supersymmetry breaking and to show that there are strong cosmological constraints on scenarios in which supersymmetry breaking vanishes in the limit in which the Planck mass is taken to infinity with some "intermediate" scale fixed. For instance, all scenarios based on gaugino condensation in a hidden sector [5] are of this type, as are all models where supersymmetry breaking is driven by $\mathrm{F}$ terms of string theory moduli.

We wish to provide a taxonomy of dynamical SUSY breaking models in which the only explicit mass scale is the Planck scale. They must explain the $M_{W} / M_{P}$ hierarchy, and be consistent with low energy phenomenology (including a small or vanishing cosmological constant, achieved through fine-tuning). The task is complicated by there being few completely generic features to analyze. The only feature apparently common to all models of dynamical SUSY breaking is the existence of an R symmetry which is either exact or nearly exact just above the scale of SUSY breaking, and which is spontaneously broken. This is not a theorem, but no known counter-examples exist [4], and there does exist an argument that generic theories without a continuous $\mathrm{R}$ symmetry will not dynamically break supersymmetry [6]. If $\mathrm{R}$ symmetry is exact, however, then it is impossible to add a constant to the superpotential to fine tune the cosmological constant. Thus viable dynamical SUSY breaking models must have a spontaneously broken, approximate R symmetry. This results in a pseudo Nambu-Goldstone boson - the R-axion - which can cause phenomenological or cosmological problems which must be addressed. 
To proceed further it is useful to distinguish between Visible Sector (VS) models of dynamical SUSY breaking - in which Planck scale physics plays no role-and Hidden Sector (HS) models which make use of nonrenormalizable operators suppressed by powers of $M_{P}$. In VS models [7] the fields whose auxiliary components are responsible for the primary breaking of SUSY have renormalizable interactions with the fields of the standard model. In extant examples the SUSY breaking sector contains fields which transform nontrivially under $S U(3) \times S U(2) \times U(1)$, and these gauge symmetries communicate SUSY breaking to the low energy world. Such models have several attractive features. They do not depend on Planck scale physics, and, modulo computational difficulties involved in solving the strongly coupled SUSY breaking sector, they give complete predictions for the parameters of the supersymmetric standard model (SSM). In particular, since SUSY breaking is communicated to the standard model via gauge interactions, squarks are automatically nearly degenerate, and there are no problems with flavor changing neutral currents. Finally, gauginos have relatively large, phenomenologically acceptable masses.

Known viable VS models have a rather complicated structure which is necessary to give the unwanted R-axion a sufficiently large mass to be compatible with bounds from experiment and astrophysical observations. Furthermore, in existing VS models, the SUSY breaking vacuum is only metastable.

In HS models, the SUSY breaking sector communicates with the standard model only via nonrenormalizable interactions suppressed by powers of $M_{P}$. Among these there is a further dichotomy: the HS may break SUSY and have a stable vacuum in flat space and only require nonrenormalizable operators to communicate SUSY breaking to the visible world; or SUSY breaking may itself arise from Planck scale nonrenormalizable interactions. The former models, where SUSY breaking occurs due to renormalizable couplings, we denote RHS, while models which require nonrenormalizable operators for SUSY breaking are referred to as NRHS models.

In section II of this paper we study NRHS models. It is not clear whether there are any explicit examples of models of this class which really have stable SUSY breaking vacua. The "racetrack" models [8] represent the state of the art. Our analysis assumes the existence of such a vacuum, and the phenomenological requirement (discussed below) that singlet $\mathrm{F}$ terms be generated, but no other specific features of gaugino condensation models.

We first analyze the relation between the scales of breaking of supersymmetry and $\mathrm{R}$ symmetry, and argue that the latter must be higher than the former if the cosmological 
constant is to vanish. In fact, the scaling is precisely right if we assume that the strong dynamics at the intermediate scale $M_{R}$ (the SUSY breaking scale is $M_{S} \sim \sqrt{M_{R}^{3} / M_{P}}$ ) spontaneously breaks all $\mathrm{R}$ symmetries down to at most a $Z_{2}$ "R-parity". This is an aesthetic argument in favor of NRHS models. In theories for which there is a larger $\mathrm{R}$ symmetry down to a scale which is below $M_{S}^{\frac{2}{3}} M_{P}^{\frac{1}{3}}$, the cosmological constant cannot be set to zero even by fine tuning.

We find however that there is a very general cosmological constraint on NRHS models, which is very hard to satisfy. We argue that such models typically contain particles, including the R-axion, with masses of order $1 \mathrm{TeV}$ and couplings proportional to $1 / M_{P}$. Under quite general assumptions, this field dominates the energy density of the universe until the time when the energy density is $\sim\left(10^{-2} \mathrm{MeV}\right)^{4}$. The baryons and radiation that populate the universe today cannot be produced until after this epoch. Consequently, a model incorporating such particles is not compatible with the standard theory of primordial nucleosynthesis. It is also likely to be incompatible with a reasonable theory of structure formation in the universe. Therefore we conclude that our present conceptions of early cosmology rule out all NRHS models.

The problems we point out in NRHS models are essentially the same as the problems [9] with the Polonyi field in the earliest hidden sector models [10]. In that context it has been suggested that the problem can be resolved by raising the gravitino and scalar masses well above the weak scale 11, 12]. In our effective Lagrangian analysis, the only mechanism available for raising the masses of the dangerous scalar fields is to make the dimensionless coefficients of certain nonrenormalizable couplings very large. The general rules of effective field theory suggest that the real meaning of large dimensionless coefficients is the breakdown of effective field theory at a scale lower than one had originally assumed. In other words, it suggests that the physics responsible for SUSY breaking is operative at a scale below the Planck mass and that we are not really dealing with a NRHS model at all.

We conclude Section III by pointing out that string theory is replete with particles that may suffer from cosmological problems of the type we have discussed here. These are the moduli fields, which exist in all $(2,2)$ string vacua, and all known $(2,0)$ models. We conclude that either one must find string vacuum states without moduli, or that strong interactions at a scale of $10^{15} \mathrm{GeV}$ or greater must give mass to the moduli fields. Since this is well above the scale of SUSY breaking (with any conjectured mechanism) we conclude that nonperturbative effects not associated with SUSY breaking must be an important part of the stringy description of the world. 
After discussing the cosmological problems of NRHS models, in Section III we show that it is possible to construct viable RHS models - hidden sector models with flat space SUSY breaking. We note however that, assuming a minimal spectrum in the visible sector, any such theory which also allows gauginos to get weak scale masses will have to invoke "supernaturalness": certain terms in the superpotential which are not forbidden by any symmetry must be assumed absent. String theoretic vacuum states often contain "stringy symmetries" Supersymmetric nonrenormalization theorems then imply that these terms are absent to all orders in perturbation theory.

We conclude that if our cosmological constraints cannot be evaded, there are only two known viable scenarios of dynamical SUSY breaking: the VS and RHS models. Both require the existence of a strongly coupled theory which breaks SUSY in flat space. The two mechanisms differ in the medium via which SUSY breaking is transmitted to the SSM, gravity or gauge interactions. As a consequence, the fundamental scale of SUSY breaking in the two classes of models differs by a factor of order $10^{4}-10^{7}$. As mentioned above, all known models of dynamical SUSY breaking possess R-axions. For VS models, these axions could be seen in laboratory experiments, or could affect stellar cooling rates and supernova dynamics. This problem was solved in [7] by the introduction of a second, slightly weaker, gauge interaction which explicitly breaks the R-symmetry via the anomaly. For RHS models, the R-axion has a decay constant of just the right size to fit into the conventional invisible axion window [13] and could be used to solve the strong CP problem. In the next section we explicitly construct an example of a model in which this possibility is realized. It requires the imposition of an anomalous $\mathrm{U}(1) \mathrm{R}$ symmetry, as well as the absence of several terms which are not forbidden by any symmetry. Unfortunately, such a model seems to be incompatible with fine tuning the cosmological constant to zero. An alternative (and perhaps more plausible), scenario for the fate of the R-axion in RHS models, is that nonrenormalizable Planck scale couplings give the axion a mass greater than or equal to the weak scale and effectively eliminate its effects on both cosmology and the strong CP problem.

1 Technically, these are higher spin chiral algebras on the world sheet. The most well known of these is the left handed world sheet $\mathrm{N}=2 \mathrm{SUSY}$ algebra of $(2,2)$ ground states. 


\section{NRHS: Hidden sector models which are supersymmetric in the flat space limit}

\subsection{The ubiquity of singlets}

A generic feature of HS models is the existence of gauge singlet superfields which communicate SUSY breaking to the visible world via interactions suppressed by powers of $M_{P}$. The requirement that such fields be gauge singlets arises from the need in the SSM to generate both dimension 3 operators with coefficients of order the weak scale, such as trilinear scalar couplings and gaugino and higgsinol masses, as well as dimension 2 operators (eg, squark masses), with coefficients of order the weak scale squared.

Squark masses will always be of the form

$$
m_{\tilde{q}}^{2} \sim \frac{\langle F\rangle^{2}}{M_{P}^{2}}
$$

where $\sqrt{\langle F\rangle}$ has dimensions of mass and is the scale of SUSY breaking in the hidden sector. In order to explain the gauge hierarchy, one must have $\langle F\rangle \lesssim M_{P} \times(1 \mathrm{TeV})$. The coefficients of dimension 3 operators, such as the gluino mass, for example, are of the form

$$
m_{\tilde{g}} \sim\left(\frac{\langle F\rangle}{M_{P}}\right)\left(\frac{\langle F\rangle}{M_{P}^{2}}\right)^{n}
$$

The gluino mass must be of the same order in $\langle F\rangle / M_{P}^{2}$ as the squark mass, or else it is essentially massless at tree level. Thus a large gluino mass requires $n=0$ in (2.1) and therefore arises from a dimension five operator,

$$
\frac{\mathcal{O}(1)}{M_{P}} \int d^{2} \Theta Z W_{\alpha}^{2}
$$

where $W_{\alpha}$ is the color superfield and $Z$ is the agent of SUSY breaking. Consequently, $Z$ must carry zero charge under any unbroken symmetry of the theory, although it may shift under an R-symmetry 3 .

2 We do not allow for the possibility of putting in weak scale supersymmetric dimension 3 terms such as the mu parameter by hand, but assume they must be generated by the same physics which breaks supersymmetry.

3 The only known models which escape this conclusion add extra chiral superfields to the visible sector, such as a color octet and some weak triplets, and require that hypercharge be unified into a nonabelian symmetry at a scale below $10^{10} \mathrm{GeV}$ [14]. 
There is still no conclusive evidence for the necessity of tree-level dimension 3 operators in the SSM, since there may remain a small window for a light gluino whose mass is generated radiatively [15]. However, it should be noted that versions of the SSM without dimension 3 operators must contain gauge singlets in the low energy visible sector in order to break an effective $R$ symmetry that would forbid radiative gluino masses and revive the old $U(1)$ problem of a light $\eta$ meson. Therefore any HS theory of dynamical SUSY breaking must contain a gauge singlet superfield. The phenomenological window for a light gluino is small and a light gluino should either be found or ruled out in the near future.

In this paper we will consider the consequences of HS models which contain a set of singlet chiral superfields $z^{A}$ in the hidden sector. First we examine NRHS models in which the $z^{A}$ singlet fields are coupled to a strongly coupled hidden sector with dynamical mass scale $M_{R}$ via nonrenormalizable interactions of the form

$$
\delta \mathcal{L}=c_{A_{1} \ldots A_{N}} \frac{z^{A_{1}}}{M_{P}} \ldots \frac{z^{A_{N}}}{M_{P}} \mathcal{O}^{4}
$$

where $\mathcal{O}^{4}$ are operators of dimension 4 in the strongly coupled theory. In the absence of these couplings, the strongly coupled theory is assumed to have a stable supersymmetric vacuum. If such operators exist at dimension 5, then the SUSY breaking scale in the visible sector will then be of order $m_{3 / 2} \sim M_{R}^{3} / M_{P}^{2}$ - one power of $1 / M_{P}$ arising from the operator (2.3), and another from the gravitational communication of SUSY breaking to the visible sector. We will also assume that, like the dilaton and moduli superfields in string theory, these fields have no renormalizable couplings in the fundamental Planck scale Lagrangian. It is important to our analysis that the fields $z^{A}$ are always small (relative to $\left.M_{P}\right)$ at the minimum of their effective potential, which we will assume to violate SUSY. Conventional field theoretic dimensional analysis depends on the assumption that the vacuum expectation values of fields are much smaller than the scale which characterizes irrelevant interactions.

The only extant examples of models which might belong to the class defined above, are the "racetrack" models of competitive gaugino condensation [8]. The models are derived from string theory and the origin of field space is taken to be a point which is natural from the string theoretic point of view. It is a distance of order $M_{P}$ from the minimum. The use of an effective Lagrangian over such a large expanse of field space is justified by an appeal to knowledge of the underlying short distance physics. Our analysis of these models would begin by reexpanding the effective Lagrangian around the minimum. We are not trying to 
establish the existence of a SUSY breaking minimum in a particular model, but rather to demonstrate some very general consequences of the existence of such a minimum.

We are now in a position to write down the effective Lagrangian for the scalar fields $z^{A}$ which results from integrating out the strongly interacting fields which get mass at the scale $M_{R}$. Since the theory at $M_{R}$ does not violate SUSY, the effective theory below the scale $M_{R}$ has the conventional supergravity form with effective potential

$$
V=e^{\frac{K(z, \bar{z})}{M_{P}^{2}}}\left(\left(K^{-1}\right)_{B}^{A}\left(D_{A} W D^{B} \bar{W}\right)-\frac{3}{M_{P}^{2}}|W|^{2}\right)
$$

where

$$
D_{A} W=W_{A}+\frac{K_{A}}{M_{P}^{2}} W
$$

and subscripts denote differentiation with respect to $z^{A}$. By convention, we have chosen $z^{A}=0$ to be the minimum, and we expand the Lagrangian in a power series around the origin. We will choose a Kahler gauge for which all holomorphic polynomials in $z$ have been shifted from the Kahler potential $K$ into the superpotential $W$. The leading nontrivial term in the Kahler potential, is of order $z \bar{z}$ with dimensionless coefficients. By linear field redefinitions, we can always bring it to the form $z^{A} \bar{z}^{A}$. The corrections to this canonical form may be written

$$
\begin{aligned}
K= & \frac{1}{2} \bar{z}_{A} z^{A}+\frac{1}{2} \frac{K_{A B}^{C}}{M_{P}} z^{A} z^{B} \bar{z}_{C} \\
& +\frac{1}{3 M_{P}^{2}} K_{A B C}^{D} z^{A} z^{B} z^{C} \bar{z}_{D}+\frac{1}{8 M_{P}^{2}} K_{A B}^{C D} z^{A} z^{B} \bar{z}_{C} \bar{z}_{D}+\text { h.c. }+\ldots
\end{aligned}
$$

The dimensionless coefficients in this expansion are functions of $M_{R} / M_{P}$ which should approach finite numbers in the limit $M_{R} \ll M_{P}$.

Similarly, the effective superpotential has an expansion:

$$
W=M_{R}^{3}\left(\omega+\omega_{A} \frac{z^{A}}{M_{P}}+\omega_{A B} \frac{z^{A} z^{B}}{M_{P}^{2}}+\ldots\right)
$$

The power of $M_{R}^{3}$ in front of this term reflects our assumption that strong dynamics at the scale $M_{R}$ are responsible for generating this superpotential. The $M_{P}$ scaling of terms that depend on the fields reflects the fact that the singlets couple only through terms of the form (2.3). Again, we expect the dimensionless coefficients to be of order one. It is conceivable that some of them are much smaller than one. For example, our strongly coupled sector might have several scales, with different symmetries broken at different 
scales. If $M_{R}$ is the largest scale at which a superpotential is generated, the complications of the strongly coupled sector would show up as anomalously small dimensionless coefficients. Another way to generate small coefficients is to assume that some of fields $z^{A}$ couple through operators of dimension higher than those in (2.3). This would make some of the coefficients positive powers of $M_{R} / M_{P}$. On the other hand, there is no natural way to make the dimensionless coefficients in this Lagrangian large. Indeed, the typical meaning of a large dimensionless coupling in an effective Lagrangian is that we have made an error in identifying the scale of high energy physics which is being ignored in the effective field theory. If the high energy scale is in fact lower than we had imagined, we naturally obtain large coefficients. In the present context, this means that the Lagrangian (2.3) is induced by physics below the Planck scale, in contradiction to our initial hypothesis.

\subsection{R-symmetry and the cosmological constant}

We now impose the constraint that our Lagrangian has a SUSY violating minimum at $z^{A}=0$, with zero cosmological constant. SUSY violation is ensured by the fact that $\omega_{A} \neq 0$. The constraint that the cosmological constant is zero is

$$
\omega_{A} \bar{\omega}^{A}-3|\omega|^{2}=0
$$

Note that this is a cancellation between numbers that are a priori of order one. In a more general $N=1$ supergravity theory, the cancellation of the cosmological constant implies that the value of the superpotential at the minimum be related to the SUSY breaking F term by $W \sim M_{P} F \equiv M_{P} M_{S}^{2}$. A nonzero value for the superpotential breaks any $\mathrm{R}$ symmetry of the theory larger than $Z_{2}$. A natural explanation for this general order of magnitude relation might be that the theory has an unbroken $\mathrm{R}$ symmetry (perhaps discrete), which is dynamically broken at a scale

$$
M_{R} \sim\left(M_{S}^{2} M_{P}\right)^{\frac{1}{3}}
$$

NRHS models implement this explanation dynamically if we assume that the strongly coupled hidden sector at $M_{R}$ is the agent of R-symmetry breaking (as it is in models based on gaugino condensation). On the other hand, if there is an exact R-symmetry which survives to scales much below (2.9), the cancellation of the cosmological constant is impossible. 
This observation is intriguing, but may have limited relevance for SUSY model building. Although it rules out models with exact $\mathrm{R}$ symmetries below the scale (2.9), it does not prevent the appearance of accidental $\mathrm{R}$ symmetries (in particular, the accidental $\mathrm{R}$ symmetries which are the key to all known models of dynamical SUSY breaking in flat space). So we obtain only the mildly restrictive new rule: $R$ symmetries (other than $R$-parity) in low energy SUSY models must be accidental i.e. they must follow as a consequence of other exact symmetries plus renormalizability. We can expect them to be broken by nonrenormalizable terms scaled by $M_{R}$ (or perhaps $M_{P}$ ). It is also impossible to rule out models in which all $\mathrm{R}$ symmetries are broken at a scale higher than (2.9). For example, many tree level superstring vacua have unbroken SUSY and no R symmetries, and the cosmological constant vanishes despite this. This may be viewed as a superstring miracle [16] : the constant in the superpotential, which is not forbidden by any field theoretic symmetry of these vacuum states, vanishes "by accident". In these vacua, the relation (2.9) is violated, but the cosmological constant might well turn out to be zero.

NRHS models thus enjoy the distinction of being able to explain dynamically the relation (2.9), which, although it by no means solves the cosmological constant problem, makes the vanishing of the cosmological constant a little more natural. Unfortunately, as we now explain, all NRHS models suffer from a cosmological problem which makes them unattractive candidates for models of the real world.

\subsection{Light scalars: a cosmological problem}

The condition that $z^{A}=0$ be a stationary point of our action can be written:

$$
\bar{\omega}^{A} \omega_{A B}+\omega_{B}-K_{A B}^{C} \bar{\omega}^{A} \omega_{C}=0
$$

Note that the noncanonical term in the Kahler potential can be important in satisfying this condition. We now want to examine the quadratic terms in the expansion of the potential around the stationary point. This task is somewhat simplified by the observation that the prefactor in the potential can be set equal to one, because the term it multiplies is already of quadratic order in the $z^{A}$ (as a consequence of the conditions (2.10) and (2.8)). Note however that the quadratic terms do depend on the quartic terms $K_{A B C}^{D}$ and $K_{A B}^{C D}$ in the Kahler potential. Since these are as yet unconstrained, we are free to choose them in such a way that the origin is a stable minimum with no flat directions. Thus there are no $a$ priori arguments against the existence of NRHS models. What we cannot do however is 
to arbitrarily choose the size of the mass terms for the $z$ fields. Their masses are of order $M_{R}^{3} / M_{P}^{2} \sim m_{3 / 2}$. Some of these masses might be smaller, if the dimensionless coefficients in (2.6) or (2.7) are small for the reasons discussed above. However, for those singlets which give rise to SSM gaugino masses, there cannot be a symmetry which prevents all couplings of the form (2.3). These will generically have masses of order the SUSY breaking scale in the visible sector, which is generally assumed to be of order $100 \mathrm{GeV}-1 \mathrm{TeV}$ in order to eliminate fine tuning problems at the weak scale.

The cosmological consequences of scalar fields with mass $m$ and gravitational strength interactions have been analyzed before [9, 12, 17, 18], and we briefly review the results here. In the early universe, typical thermal values of the scalar field strength are far from the origin (which we take to be the low temperature minimum of the potential), and so there is a Bose condensate. Such a condensate cannot be eliminated by inflation [19,

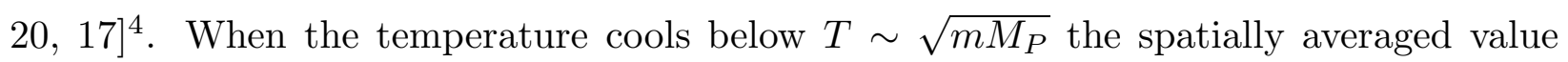
of the scalar field oscillates about the minimum, and so we have a gas of cold bosons. Assuming that the inital expectation value of the field is of order $M_{P}$, if $m$ is greater than $10^{-28} \mathrm{eV}$, these bosons contribute too much energy density to the mass of the universe to be consistent with standard cosmology, unless their lifetime $\tau$ is sufficiently short so that they decay before nucleosynthesis. However for bosons with gravitational strength interactions, $\tau$ must be greater than $\mathcal{O}\left(M_{P}^{2} / m^{3}\right)$, and once they have decayed the temperature $T_{R}$ of the universe is $\mathcal{O}\left(\sqrt{M_{P} / \tau}\right) \lesssim \sqrt{m^{3} / M_{P}}$. Standard nucleosynthesis requires that $T_{R}$ be larger than $\mathcal{O}(1) \mathrm{MeV}$, and so that the mass $m$ be larger than $\mathcal{O}(3) \times 10^{4} \mathrm{GeV}$. Since, in the models of interest, both $m$ and the weak scale are naturally less than or of order the gravitino mass, conventional nucleosynthesis requires a supersymmetry breaking scale which is uncomfortably large for a natural resolution of the gauge hierarchy problem. Furthermore, the scalar decays generate a tremendous amount of entropy, which would dilute any initial baryon abundance to well below what is observed. Unless the decays also lead to baryon number production 5 , then we must demand that $T_{R}$ is sufficiently large for baryogenesis. For instance, in order to allow for baryogenesis during the weak

4 unless the Hubble constant during the inflationary period is smaller than the mass of the scalar field. However, with such late inflation it is not possible in a natural model to reheat after inflation to a temperature above $\sqrt{m^{3} / M_{P}}$, which is below the temperature needed for nucleosynthesis if $m<1 \mathrm{TeV}$.

5 For example the scalar decays could proceed via baryon number and CP violating interactions. 
phase transition [21] $m$ and $m_{3 / 2}$ must be greater than $\mathcal{O}\left(10^{8}\right) \mathrm{GeV}$. We conclude that the cosmological constraints on NRHS models make these theories much less attractive.

\subsection{Exotica}

In the above discussion we assumed both that the HS had a stable supersymmetric vacuum in the $M_{P} \rightarrow \infty$ limit, and that the $z^{A}$ fields had no renormalizable couplings. We now briefly consider what happens if one relaxes those two conditions.

First we offer a toy example of a model in which gravitational effects could be responsible for supersymmetry breaking, but for which there is no stable vacuum as $M_{P} \rightarrow \infty$ - the example is simply massless QCD, with fewer flavors than colors. In flat space, there is a dynamically generated superpotential of the form [2]

$$
W_{\text {eff }}=\mathcal{O}(1) \Lambda^{\frac{3 n_{c}-n_{f}}{n_{c}-n_{f}}}\left(\frac{1}{\operatorname{det}_{i j} \bar{q}_{i}^{a} q_{j}^{a}}\right)^{\frac{1}{n_{c}-n_{f}}}
$$

where $i, j$ are flavor indices, $a$ is a color index, $\Lambda$ is the QCD scale and $n_{c}$ and $n_{f}$ are the number of colors and flavors respectively. This superpotential drives the squark vevs to infinity and the theory has no ground state. However if one naively includes the effects of supergravity by using the effective potential (2.4), assuming a Kahler potential of form (2.6), then one finds that the vevs could be stabilized at a value of order $M_{P}$ (where, however, an effective field theory analysis breaks down) and the supersymmetry breaking scale is then

$$
M_{S} \sim\left(\frac{\Lambda^{3 n_{c}-2 n_{f}}}{M_{P}^{n_{c}}}\right)^{\frac{1}{2\left(n_{c}-n_{f}\right)}} .
$$

Although because of the large field strengths there is no reliable way of computing whether or not gravitational effects actually stabilize this theory and break supersymmetry, one can see using dimensional analysis that even if this is the case, there will be a light scalar particle of mass $M_{S}^{2} / M_{P} \sim m_{3 / 2}$, with gravitational strength couplings to other light fields. Because this theory has a spontaneously broken exact $\mathrm{U}(1) \mathrm{R}$ symmetry, the R-axion is a massless Goldstone boson, with decay constant of order $M_{P}$.

Next we consider models in which the $z^{A}$ fields have renormalizable couplings to other fields, and which might also produce a SUSY breaking scale vanishing as $M_{P} \rightarrow \infty$. The generic example is of the form

$$
c_{A} z^{A} R \bar{R}
$$


where $R$ is some representation of the strongly coupled hidden sector gauge group, or of the visible sector gauge group. We assume this strongly coupled theory has a stable, supersymmetric vacuum to fit in the NRHS category of models. First consider the $M_{P} \rightarrow$ $\infty$ limit. On intregrating out the strongly interacting fields $R$, the scale $M_{R}$ will generically be induced in the effective Kahler potential for the singlets $z^{A}$. If at the scale $M_{R}$ a direct effective superpotential for the fields $z^{A}$ is not generated, then the scalar potential is flat and only Planck scale effects could possibly break SUSY. For instance, when Planck scale effects are included and the scalar potential (2.4) is expanded in inverse powers of $M_{P}$, the effective superpotential for the singlets below the scale $M_{R}$ of the strongly coupled physics could be of the form

$$
\frac{M_{R}^{3}}{M_{P}} z_{A}
$$

which would give SUSY breaking at a scale $\sqrt{M_{R}^{3} / M_{P}}$. The resulting theory has a light particle, but it could receive a radiatively generated mass of order $M_{R}^{2} / M_{P} \sim\left(m_{3 / 2}^{2} M_{P}\right)^{1 / 3}$ rather than $m_{3 / 2}$ (which we have shown is generic in NRHS models), and it can have couplings which are suppressed by $M_{R}$ rather than $M_{P}$. The origin of this relatively large contribution to the scalar masses is a noncanonical term in the Kahler potential of the form

$$
K_{A B}^{C} z^{A} z^{B} \frac{\bar{z}_{C}}{M_{R}}
$$

induced by the strong interaction between the scalars and the hidden sector. We regard this class of models as somewhat special, since if we assume, in accord with all existing evidence, that nonperturbative effects generate any superpotential consistent with the symmetries of the model, then it is very hard to satisfy the condition italicized above. Indeed, we have so far been unable to find any SUSY breaking models in this class in which all mass scales below the Planck mass are generated dynamically, and which is natural in the sense that all terms consistent with the symmetries are included. Thus, although this class of NRHS models may evade our cosmological bound, it does not seem attractive. It is concievable that the class is in fact empty.

\subsection{Implications for string theory}

Brustein and Steinhardt have recently pointed out cosmological maladies of superstring inspired models even more severe than those we have discussed here [22]. These difficulties involve specific properties of the dilaton superfield and are a cosmological version of the Dine-Seiberg problem [23]. The constraints on NRHS models that we have 
discovered are much more general. They do not assume that the dilaton has anything to do with SUSY breaking, and apply equally well to NRHS models which have nothing to do with string theory.

Note that if, as suggested by our constraints, the mechanism which fixes the dilaton vacuum expectation value does not break SUSY, the Brustein-Steinhardt problem is more easily resolved 6 . For instance, the dynamics which stabilizes the vevs of the dilaton and other moduli could be strong at a scale not far below $M_{P}$, leading to a large barrier between the desired ground state and the disastrous vacuum at infinite dilaton VEV. For instance if the moduli are stabilized by strong dynamics at a scale of $\sim 10^{16} \mathrm{GeV}$, they could give rise to natural inflation at a scale which gives the desired primordial density fluctuations [25], with subsequent reheating to $10^{10} \mathrm{GeV}$. In any case, for the universe to be at least at a temperature of $1 \mathrm{MeV}$ (as necessary for nucleosynthesis) after the moduli decay, they should be stabilized by dynamics above $10^{14} \mathrm{GeV}$. Thus, there appears to be a general discrepancy between the scale of supersymmetry breaking required by phenomenology and the mass scale for moduli fields required to avoid the cosmological difficulties of light weakly coupled bosons. It appears that a consistent string theoretic description of nature requires nonperturbative dynamics at a scale higher than than of SUSY breaking, or the existence of string vacua without moduli.

Finally, let us add to our catalogue of mechanisms for dynamical SUSY breaking the still mysterious proposal of "stringy nonperturbative breaking" [26] Since SUSY breaking must occur well below the Planck scale, these effects must be encodable in a superpotential in a supersymmetric low energy effective lagrangian just above the SUSY breaking scale. Since the physical states contributing to these effects all have masses of order the string scale [26], we expect a contribution to the superpotential of the form

$$
\epsilon M_{S}^{3} W\left(\frac{z_{i}}{M_{S}}\right)
$$

where $z_{i}$ are some chiral superfields, and $M_{S}$ is the string scale, which is only slightly smaller than the Planck mass. $\epsilon$ is the small parameter $\left(\sim e^{-\frac{1}{g}}\right)$ which identifies this as a nonperturbative effect in string theory. This is essentially the same kind of potential that we analyzed above and it leads to the same kind of problems. Our ignorance of the nature (and even the existence) of stringy nonperturbative SUSY breaking is so great that we should take this argument with a grain of salt, but one should certainly worry that this hypothetical mechanism for SUSY breaking may encounter cosmological problems.

6 An alternative solution based on the ideas of chaotic inflation has been suggested by Shenker [24]. 


\section{RHS: Hidden sector models which dynamically break SUSY in the flat space limit}

Having discussed NRHS models in which SUSY is restored in the $M_{P} \rightarrow \infty$ limit, we turn to RHS models where gravity serves only to communicate SUSY breaking. We find that while RHS models can easily evade the cosmological problems found for NRHS scenarios, it is difficult to give the visible gauginos a weak scale mass even with the introduction of gauge singlets. We do explicitly construct a model, where there is a hidden sector contribution to gaugino masses which is suppressed relative to the squark and slepton masses by a loop factor.

It has been known for some time that dynamical supersymmetry breaking is possible in a renormalizable field theory in flat space [3, 4], and that it is difficult to incorporate this mechanism into a realistic model. An example of flat space dynamical supersymmetry breaking in the visible sector is given in [7]. The main problem with RHS models is that the gauginos in the visible sector get only a very small mass [4]. One alternative, with greatly expanded visible sector, is described in ref. [14]; here we will assume that the visible sector has minimal field content. Another alternative is that the gauginos gain mass only from loops involving visible sector particles (and from electroweak symmetry breaking) [15], in which case evidence for light gluinos $(\sim 500 \mathrm{MeV})$ or charginos $(\sim 50 \mathrm{GeV})$ could appear soon. If larger gaugino masses are required, then the hidden sector must contain a gauge singlet as discussed in section 2, with a coupling to the visible gauge fields as in eq. (2.2). This singlet must also couple to the hidden supersymmetry breaking sector with renormalizable couplings, since in order to give the gauginos a weak scale mass its $\mathrm{F}$ component must be of order the supersymmetry breaking scale squared. However existing models of flat space dynamical supersymmetry breaking only contain matter superfields in chiral representations of the gauge group, and so can couple to a gauge singlet only via terms of dimension five or more. Thus the simplest possible strategy, which is to take for the hidden sector a model of dynamical supersymmetry breaking with matter fields in purely chiral representations, leads to very light gauginos in the visible sector.

In order to give gauginos a larger mass, we need to find a model of flat space dynamical supersymmetry breaking in which some matter superfields are in real representations of the gauge group, so that they can couple to a gauge singlet via renormalizable interactions. Such models are not impossible to find. An example was given in [7], but this model has the drawback of having a supersymmetric minimum at infinity in field space, as well as a 
local supersymmetry breaking minimum. Here we will give an example of such a model which probably has a unique supersymmetry breaking ground state.

Many dynamical supersymmetry breaking models contain global symmetries, which we can weakly gauge without affecting the supersymmetry breaking dynamics. Our strategy is to take such a model, and to then add superfields in real representations of this new weak gauge symmetry, which can also couple to a the gauge singlet. For example, we can take SU(7) for the "supercolor" gauge group, whose dynamics are responsible for breaking supersymmetry, with matter superfields in the representation $\mathbf{2 1} \oplus 3 \overline{\mathbf{7}}$. It has been argued that this theory breaks supersymmetry and has a stable ground state [4] if there is a nonzero superpotential $W=\lambda_{1} \mathbf{2 1}_{a b} \overline{\mathbf{7}}_{1}^{a} \overline{\mathbf{7}}_{2}^{b}$, where $a, b$ are $\mathrm{SU}(7)$ indices. An SU(2) global symmetry is preserved by this superpotential, which we weakly gauge, requiring that we add an odd number of SU(2) doublets to the theory to cancel the SU(2) anomaly. The minimal set of matter fields, which will allow us to couple a gauge singlet to the supersymmetry breaking sector via renormalizable interactions, transforms under $\mathrm{SU}(7) \otimes \mathrm{SU}(2)$ as

$$
(\mathbf{2 1}, \mathbf{1}) \oplus(\overline{\mathbf{7}}, \mathbf{2}) \oplus(\overline{\mathbf{7}}, \mathbf{1}) \oplus 3(\mathbf{1}, \mathbf{2}) \oplus(\mathbf{1}, \mathbf{1})
$$

For a superpotential we take

$$
W_{\text {hidden }}=\lambda_{1} \epsilon^{i j}(\mathbf{2 1}, \mathbf{1})_{a b}(\overline{\mathbf{7}}, \mathbf{2})_{i}^{a}(\overline{\mathbf{7}}, \mathbf{2})_{j}^{b}+\lambda_{2} \epsilon^{i j}(\mathbf{1}, \mathbf{2})_{1 i}(\mathbf{1}, \mathbf{2})_{2 j}(\mathbf{1}, \mathbf{1})
$$

where $i, j$ are $\mathrm{SU}(2)$ indices. Unfortunately the ground state of this model is strongly coupled [4], and we cannot reliably compute its features. However it is at least plausible that supersymmetry breaking induces a negative mass squared for the scalar components of the $(\mathbf{1}, \mathbf{2})$ representations, and induces expectation values for them. We will also assume that nonsupersymmetric quartic terms are induced, which stablize the expectation values at finite values. Then the $\mathrm{F}$ term for the gauge singlet will be nonzero.

In order to communicate supersymmetry breaking to the gauginos in the visible sector, we add to the Lagrangian a term (2.2), where $Z$ is now the gauge singlet field $(\mathbf{1}, \mathbf{1})$. Supergravitational interactions will induce masses for the scalar fields of the visible sector which are of order the gravitino mass. Since the F term for the singlet, which is responsible for the visible gaugino masses, is only induced at one loop by the weak $\mathrm{SU}(2)$ gauge coupling, the gaugino masses will be much smaller than the supersymmetry breaking scalar masses, by a factor of order $\alpha_{\mathrm{SU}(2)} / \pi$. Therefore this model has rather light gauginos, 
although the hidden sector contribution to their mass is larger than the contribution arising from loops in the visible sector.

The hidden sector of this model has an exact nonanomalous $U(1) R$ symmetry, which is spontaneously broken at the supersymmetry breaking scale, and hence has an R-axion. It is possible to arrange the couplings to the visible sector such that the $\mathrm{R}$ symmetry is broken only by a color anomaly, and the R-axion is just the usual axion which solves the strong CP problem. Remarkably, a gravitino mass of order the weak scale results from a supersymmetry breaking scale of $\sim 10^{11} \mathrm{GeV}$, which fits precisely into the allowed window for the invisible axion decay constant. The singlet field $Z$ must have $\mathrm{R}$ charge zero, so that the coupling (2.2) is allowed, and we take the Higgs fields of the visible sector to also have $\mathrm{R}$ charge zero, which forbids a direct supersymmetric mass term. If we then take the Kahler potential to include terms

$$
\int d^{4} \theta \frac{\mathcal{O}(1)}{M_{P}} Z^{*} H_{1} H_{2}+\frac{\mathcal{O}(1)}{M_{P}^{2}} H_{1} H_{2}\left(\sum_{n} s_{n} s_{n}^{*}\right)
$$

where the $s_{n}$ are generic hidden sector superfields, we will induce a small higgsino mass term (a " $\mu$ term") of order the gaugino mass, as well as a larger supersymmetry breaking mass term for the scalar components of the Higgs. Thus this model has several desirable features: all mass scales other than the Planck mass are generated dynamically, the " $\mu$ problem" is solved by the mechanism of Guidice and Masiero [27], and there is no strong $\mathrm{CP}$ problem. One still must worry about electric dipole moments which could be induced by $\mathrm{CP}$ violation in the terms in eq. (3.3), (in the minimal supersymmetric standard model the relative phase between these two terms corresponds to the phase which is usually called $\phi_{B}$, which induces quark and lepton dipole moments at one loop). However, these dipole moments are suppressed by the small size of the gaugino and higgsino mass terms relative to the squark and slepton masses, and can be within experimental bounds for phases of order one.

Unfortunately there are several troubling naturalness problems with any model of this type. For one thing the superpotential (3.2) is not the most general allowed by the symmetries. This is a general problem, as can be seen by the following argument. Because of the coupling (2.2), the singlet field cannot carry any non-zero charges except a shift symmetry $Z \rightarrow Z+i$ constant, and we also need the singlet to have a renormalizable coupling to the supersymmetry breaking sector, e.g.

$$
Z \bar{R} R
$$


where $R$ and $\bar{R}$ are superfields coupled to the supersymmetry breaking sector. There is no symmetry which allows the couplings (2.2) and (3.4) which does not also allow a coupling $\bar{R} R$, and so $R$ and $\bar{R}$, which are responsible for communicating supersymmetry breaking to the singlet, naturally would have mass of order $M_{P}$, which would greatly suppress their ability to communicate supersymmetry breaking to the singlet. It is therefore necessary to invoke "supernaturalness", i.e. the fact that in supersymmetric theories it is technically natural to omit arbitrary terms from the superpotential which are allowed by all symmetries of a theory, and this does not destroy the renormalizability of the theory. For instance, such missing terms often arise in string theories. Still, we find it unsatisfying to have to rely on unknown Planck scale physics to explain the absence of undesirable terms. An even worse feature of this model is that the R symmetry which solves the strong CP problem is imposed by hand, and is not an accidental consequence of other symmetries of the theory. In the previous section we argued that we cannot require any $\mathrm{R}$ symmetries (other than accidental ones) to be unbroken below a scale $M_{R} \sim M_{S}^{\frac{2}{3}} M_{P}^{\frac{1}{3}}$, or it will be impossible to tune the cosmological constant to zero. Of course, since one has to rely on supernaturalness anyway to suppress unwanted terms, one need not invoke any R symmetry, but then there is no reason not to expect the axion to gain mass from Planck scale physics of order $M_{S}^{n+1} / M_{P}^{n}$, i.e. the weak scale for $n=1$. Unless $n$ is larger than 6 , such an axion will play no role in solving the strong $\mathrm{CP}$ problem although it always either decays quickly enough or contributes a small enough mass density to the universe to escape all cosmological problems.

In summary, dynamical supersymmetry breaking in flat space is a viable option for hidden sector models, although one which will typically lead to light gauginos in the visible sector. The simplest possibility is that the hidden sector model has purely chiral matter field content, and so visible gaugino masses only arise from loops in the visible sector and are very small (e.g. $\sim 500 \mathrm{MeV}$ for the gluino). Somewhat larger gaugino masses are possible if one complicates the hidden sector to allow for a gauge singlet which has renormalizable couplings with the supersymmetry breaking sector. Any such model requires the omission of some terms in the effective Lagrangian which are not forbidden by any symmetry. In explicit models which we have constructed, the gaugino masses are still suppressed relative to the scalar masses by a weak hidden sector gauge coupling. Such models generically contain an R-axion with a decay constant equal to the SUSY breaking scale-this could be identified with the invisible axion, and in general presents no cosmological problems. 


\section{Summary}

There are many possible scenarios for solving the gauge hierarchy problem via dynamical supersymmetry breaking. Here we have shown that models in which Planck scale effects give rise to dynamical supersymmetry breaking typically contain light (less than 1 $\mathrm{TeV}$ ) scalars with gravitational strength couplings. For instance all string inspired models, where supersymmetry breaking is driven by the same dynamics which stabilizes the moduli, have such light, weakly coupled states. These are difficult to fit into an acceptable cosmological scenario even when inflation is taken into account. More promising are models in which supersymmetry is dynamically broken in flat space. Hidden sector models with flatspace supersymmetry breaking typically produce very small masses for the visible gauginos. Increasing the gaugino masses in such models requires either a greatly expanded visible sector, or the introduction of a singlet in the invisible sector with renormalizable couplings and the omission of some terms which are not forbidden by any symmetry. Furthermore the gaugino masses are still suppressed compared with squark and slepton masses. Thus in all known viable hidden sector models, the gauginos could be discovered soon. Models in which supersymmetry is broken dynamically in the visible sector can be constructed which have no difficulties in producing weak scale gaugino masses, which explain the absence of flavor changing neutral currents in a simple way, and which present no phenomenological or cosmological difficulties. We conclude that the latter class of models deserves further study.

Acknowledgements: We are grateful to Nathan Seiberg for many useful comments and critcisms. A.N. and D.K. thank the theory goups at Rutgers and at CERN for their hospitality. 


\section{References}

[1] E. Witten, Nucl. Phys. B188 (1981) 513

[2] I. Affleck, M. Dine and N. Seiberg, Nucl. Phys. B241 (1984) 493; G.C. Rossi, G. Veneziano, Phys. Lett. 138B (1984) 195

[3] I. Affleck, M. Dine and N. Seiberg, Phys. Rev. Lett. 52 (1984) 1677; Y. Meurice and G. Veneziano, Phys. Lett. 141B (1984) 69

[4] I. Affleck, M. Dine and N. Seiberg, Nucl. Phys. B256 (1985) 557

[5] Recent reviews include D. Amati et al., Phys. Rep. 162 (1988) 169; H.-P. Nilles, Int. J. Mod. Phys. A5 (1990) 4199; J. Louis, in Proceedings of the 1991 DPF meeting in Vancouver, (World Scientific, 1992)

[6] A.E. Nelson and N. Seiberg, in preparation.

[7] M. Dine and A.E. Nelson,Phys. Rev. 48 (1993) 1277.

[8] N.V. Krasnikov, Phys. Lett. 193B (1987) 37; L. Dixon, in Proceedings of the A.P.S. DPF Meeting, Houston, (1990), L. Dixon, V. Kaplunovsky, J. Louis, and M. Peskin, unpublished (1990), J.A. Casas et al., Nucl. Phys. B347 (1990) 243; T. Taylor, Phys. Lett. 252B (1990) 59; B. de Carlos, J.A. Casas and C. Munoz, Phys. Lett. 263B (1991) 248

[9] G. D. Coughlan et al., Phys. Lett. 131B (1983) 59

[10] J. Polonyi, Budapest preprint KFKI-1977-93 (1977); E. Cremmer, S. Ferrara, L. Girardello, and A. van Proeyen, Nucl. Phys. B212 (1983) 413

[11] E. Cohen, J. Ellis, K. Enqvist and D. V. Nanopoulos, Phys. Lett. 161B (1985) 85

[12] J. Ellis, D.V. Nanopoulos, and M. Quiros, Phys. Lett. 174B (1986) 176

[13] Relevant reviews include J.E. Kim, Phys. Rep. 149 (1987) 1; R. Peccei, published in CP Violation, ed. C. Jarlskog (1988)

[14] L.J. Hall and L. Randall, Nucl. Phys. B352 (1987) 289; M. Dine and D. MacIntire, Phys. Rev. 46 (1992) 2601

[15] R. Barbieri, L. Girardello, and A. Masiero, Phys. Lett. 127B (1983) 429; R. Barbieri and L. Maiani, Nucl. Phys. B243 (1984) 429

[16] M. Dine and N. Seiberg, Nucl. Phys. B301 (1988) 357; Nucl. Phys. B306 (1988) 137

[17] G. German and G.G. Ross, Phys. Lett. 172B (1986) 305

[18] O. Bertolami, Phys. Lett. 209B (1988) 277

[19] A.H. Guth, Phys. Rev. 23 (1981) 347; A. Linde, Phys. Lett. 108BB (1982) 389; A. Albrecht and P. Steinhardt, Phys. Rev. Lett. 48 (1982) 1220

[20] A.S. Goncharev, A. D. Linde, and M. I. Vyssotsky, Phys. Lett. 147B (1984) 279

[21] For a recent review, see A.G. Cohen, D.B. Kaplan and A.E. Nelson, UCSD preprint UCSD-PTH-93-02 (1993), to be published in Ann. Rev. of Nucl. and Part. Phys., vol. 43

[22] R. Brustein and P. Steinhardt, Phys. Lett. 302B (1993) 196 
[23] M. Dine, N. Seiberg, Phys. Lett. 162B (1985) 299

[24] S. Shenker, private communication

[25] K. Freese, J.A. Frieman, and A.V. Olinto, Phys. Rev. Lett. 65 (1990) 3233

[26] S.H. Shenker, talk given at the Cargese Workshop on Random Surfaces, Quantum Gravity and Strings, Cargese, France, (1990), Published in the proceedings.

[27] G.F. Giudice and A. Masiero, Phys. Lett. 206B (1988) 480 